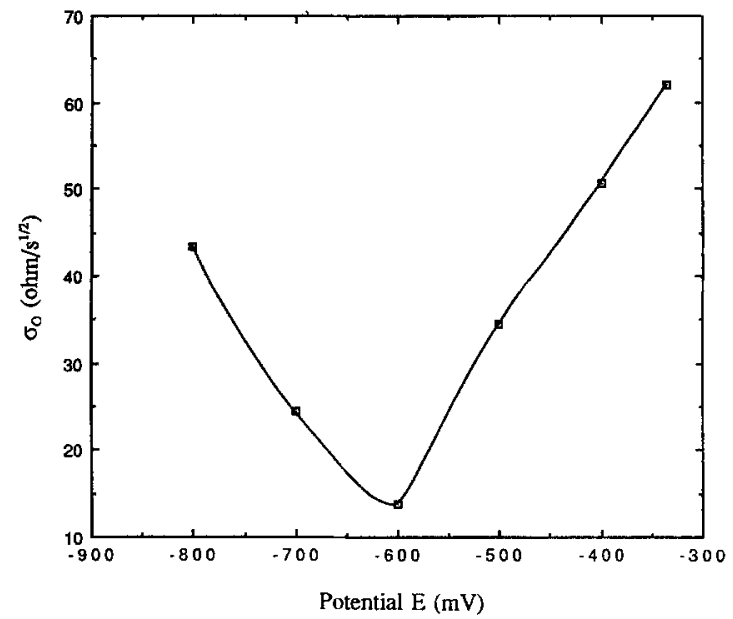

Fig. 5. Warburg coefficient-potential curve for preoxidized $\mathrm{Ni}$ in fused $\mathrm{Na}_{2} \mathrm{SO}_{4}$ at $1200 \mathrm{~K}$.

irreversible process, $\sigma_{o}$ and $R_{\mathrm{O} / \mathrm{s}}$ can be expressed as following

$$
\begin{gathered}
\sigma_{\mathrm{o}}=\frac{R T}{n^{2} F^{2} C_{\mathrm{o}}^{*} \sqrt{2 D_{\mathrm{o}}} \beta} \\
R_{\mathrm{O} / \mathrm{S}}=\frac{R T}{n^{2} F^{2} C_{\mathrm{o}}^{*} k_{\mathrm{sh}}^{\mathrm{a}} \beta \exp (-\beta \phi)}
\end{gathered}
$$

where $C_{0}^{*}$ and $D_{0}$ are the bulk concentration and the diffusion coefficient of $\mathrm{S}_{2} \mathrm{O}_{7}^{2-}$ in the melt, respectively, $\beta$ is the cathodic transfer coefficient, $k_{\mathrm{sh}}^{\mathrm{a}}$ is the rate constant of the cathodic reaction, and $\phi=(n F / R T)\left(E-E_{0}\right) . R, T, n$, and $F$ have their usual meanings. Since the salt melt was not exposed to the $\mathrm{SO}_{2}-\mathrm{O}_{2}$ gas mixture until the experiment started, the bulk concentration of $\mathrm{S}_{2} \mathrm{O}_{7}^{2-}$ (dissolved $\mathrm{SO}_{3}$ ) was low at first. As the impedance measurements proceeded with increasing polarization, the dissolution of $\mathrm{SO}_{3}$ continued and the bulk concentration of $\mathrm{S}_{2} \mathrm{O}_{7}^{2-}$ gradually increased. Thus, instead of a constant value, a decreasing $\sigma_{o}$ was observed. The changing bulk concentration of $\mathrm{S}_{2} \mathrm{O}_{7}^{2-}$, coupled with the overpotential term $\exp (-\beta \phi)$, also caused $R_{\mathrm{O} / \mathrm{S}}$ to decrease as the cathodic polarization increased, until the reaction became diffusion limited.

\section{Conclusions}

Double layer capacitance in a fused $\mathrm{Na}_{2} \mathrm{SO}_{4}$ film on a preoxidized $\mathrm{Ni}$ electrode was measured by electrochemical impedance spectroscopy. A maximum capacitance was observed at a potential of $-0.6 \mathrm{~V}$ ( vs. $\mathrm{Ag} / \mathrm{Ag}_{2} \mathrm{SO}_{4}$ reference electrode), which, based on the polarization resistance, diffusion impedance, and potentiodynamic polarization measurements, resulted from the additional contribution of a faradaic reaction. The decrease in polarization resistance and diffusion impedance with increasing polarization potential was attributed to the overpotential effect and the time-dependent dissolution of $\mathrm{SO}_{3}$. Application of the ac impedance technique is ideal in this case, because the system has been thoroughly studied (9) and an equivalent circuit unambiguously decided.

\section{Acknowledgments}

This research was sponsored by the National Science Foundation under Grant No. DMR 8620311. Critical reading of this manuscript by $R$. A. Rapp is gratefully acknowledged.

Manuscript submitted Dec. 10, 1990; revised manuscript received March 11, 1991.

The Ohio State University assisted in meeting the publication costs of this article.

\section{REFERENCES}

1. I. L. Cooper, J. A. Harrison, and D. R. Sandbach, Electrochim. Acta, 23, 527 (1978).

2. I. L. Cooper and J. A. Harrison, ibid., 29, 1147 and 1165 (1984).

3. W. Wilhelmsen and T. Hurlen, ibid., 32, 95 (1987).

4. C. Clerc and D. Landolt, ibid., 33, 859 (1988).

5. M. Weidenauer and K. G. Weil, Ber. Bunsenges. Phys. Chem., 92, 1368 (1988).

6. A. D. Graves and D. Inman, J. Electroanal. Chem., 25, 357 (1970).

7. K. R. Painter, P. Ballone, M. P. Tosi, P. J. Grout, and N. H. March, Surf. Sci., 133, 89 (1983).

8. H. Shih and H. W. Pickering, This Journal, 134, 1943 (1987).

9. Y. M. Wu and R. A. Rapp, This Journal, Accepted for publication.

10. M. Sluyters-Rehbach and J. H. Sluyters, in "Electroanalytical Chemistry," Vol. 4, A. J. Bard, Editor, Marcel Dekker, New York (1970).

\title{
Enhanced Metal Recovery in Fluidized Bed Electrodes with a Fin-Type Current Feeder
}

\author{
Shi-Chern Yen* and Ching-Yih Yao \\ Department of Chemical Engineering, National Taiwan University, Taipei, Taiwan, China
}

ABSTRACT

A rectangular side-by-side fluidized bed electrode for metal recovery from dilute copper solutions was investigated. Because the contacts between fluidized particles and current feeders are particularly important, fin-type current feeders were used to enhance the deposition rate. Polarization curves with a fin-type current feeder were measured at various Reynolds numbers. It is shown in the experiments that fin-type current feeders provide higher deposition rates for the same electrode potential than planar current feeders in fluidized bed electrodes.

Fluidized bed electrodes have been proposed since 1966 $(1,2)$. The conductive particles as electrodes are fluidized in the cell by flows of electrolytes, and electrochemical reactions proceed on electrode surfaces by applying electrical voltage to the cell. The fluidized bed electrode could result in high productivity, even in the condition of low current densities due to its high specific surface area. Because of the particle motion in fluidized bed electrodes, ag-

\footnotetext{
*Electrochemical Society Active Member.
}

glomeration due to metal deposition is avoided, unlike in packed bed electrodes $(3,4)$, and this also allows continuous removal of metals by introducing small particles at the top of the bed, with the withdrawal of grown particles at the bottom. Several potential applications have been studied (5-8), but fluidized bed electrodes are especially important in metal recovery where the main application can be expected (9-12).

Many fundamental investigations have been carried out and have concluded that the electrochemical behavior of 
fluidized bed electrodes is affected by bed expansion, bed height or bed thickness, current density, and the reactant concentration in the electrolyte $(10,11,13,14)$, and among them, bed expansion is a particularly important factor. It has been found that in the direction normal to the current feeder, the active regions are near the current feeder as well as the membrane, and there exists an anodic region in which the potential of conductive particles is higher than that of the electrolyte phase, and the region will be enlarged by increasing bed expansions (13). This phenomenon arises from discontinuity of the particle phase, in which it cannot be regarded as equipotential any more.

As a matter of fact, effective solid conductivity is the key point of electrochemical behavior in fluidized bed electrodes. Because particulate electrodes contact with each other instantaneously, the order of magnitude of the effective conductivity is the same as that of the electrolyte phase $(15,16)$. Theoretical estimation of effective conductivities is based on what mechanism of charge transfer is assumed. There are a few mechanisms proposed, and two of the most important are described briefly as follows:

1. Collision mechanism $(17,18)$.- -The electrical double layers of particles are charged while contacted with current feeders or other particles which carry different charges, and are then moved to other positions for being discharged by either sharing with other particles or by electrochemical reactions.

2. Conductive mechanism $(15,19)$.-Aggregated particles in the fluidized bed contact with others to form chains which may touch either the current feeder or other chains, and charges will pass through the chains by electronic conduction. Because the chain exists for a short time period and then is destroyed and reformed as another particle chain, potential fluctuation has been observed (20). The aggregated particle chains can be classified by whether or not they touch current feeders. The chains touching current feeders are called "monopolar," and those not touching current feeders are called "bipolar." Either reduction or oxidation reactions can occur on the surface of a monopolar particle, but both of them occur on the opposite side surfaces of bipolar particles at the same time, respectively. Lee et al. (21) have measured the bipolarity in fluidized bed electrodes. Sabacky and Evans (15), and Huh and Evans (22) have concluded that this conductive mechanism is the most probable in their experimental investigations.

In our investigations the mechanism of charge transfer is considered as a conductive mechanism, and we tried to remove the limitation of bed expansion in the applications of fluidized bed electrodes by the appropriate design of the current feeder, for example using a fin-type current feeder. Macroscopic performances of the fluidized bed electrodes with a fin-type current feeder have been carried out by measuring their polarization curves. The cell performance of cupric ion removal from dilute solutions in fluidized bed electrodes was also investigated by circulating the electrolytes through the fluidized cell. Concentration of cupric ion in the reservoir was measured with the deposition time. It was found in our measurements that the fluidized electrolytic cell with a fin-type current feeder can offer higher current densities and give broader allowance of bed expansion.

\section{Experimental}

Experimental apparatus.--The rectangular side-by-side fluidized bed cell is depicted in Fig. 1. The cell is separated into upper and lower parts. The lower part is the electrolyte entrance and flow developing region, which consists of glass beads of $1.5 \mathrm{~mm}$ diam. The upper part is the region of the electrolytic cell and is separated into two sections by an anion exchange membrane (Asahi Glass Company). The left side is the cathodic part, containing a current feeder with copper particles (Gredmann Company), sieved into the size range of $425-600 \mu \mathrm{m}$ as cathodes. The average diameter of copper particles is $512 \mu \mathrm{m}$ and the unfluidized bed porosity is 0.688 . The right side is the anodic section which is packed with platinized titanium screens, where oxygen evolution occurs. The length of both upper and lower parts is $22.5 \mathrm{~cm}$, with $3.8 \mathrm{~cm}$ in side width, and $2 \mathrm{~cm}$
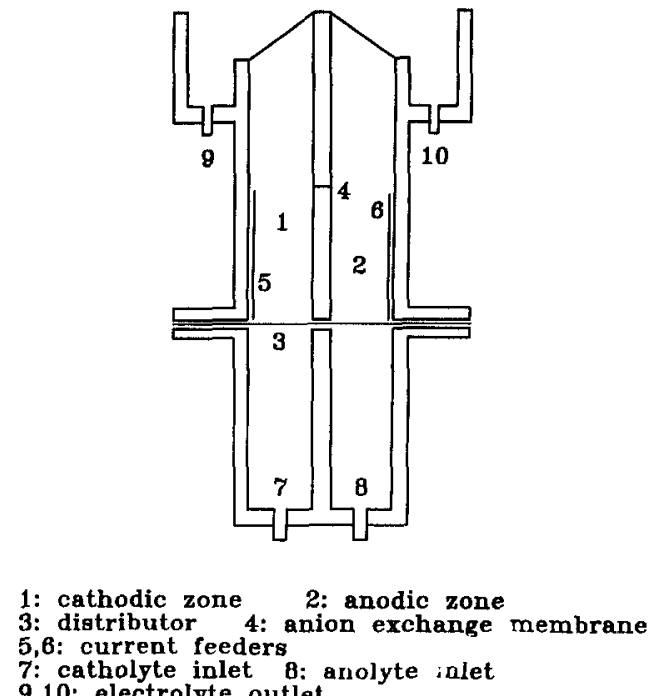

9,10: electrolyte outlet

Fig. 1. The fluidized bed cell.

in depth. Anolytes and catholytes were pumped into the cell at the lower parts respectively from both reservoirs, and the anolytes contained sodium sulfate $0.8 M$. The catholytes consisted of cupric sulfate in the range of several $\mathrm{m} M$, as well as supporting electrolytes of sodium sulfate $0.8 M$, and buffers to control $\mathrm{pH}$ in a range between 3 and 4 . The solution conductivity is $0.065(\Omega \mathrm{cm})^{-1}$. All chemicals made by Merck Company are analytical grades.

A fin-type current feeder made of titanium is shown as Fig. 2. Three different current feeders were used in the experiments. Two of them were fin types, and the length $L$ of fins was 0.7 and $1.2 \mathrm{~cm}$ respectively; they were respectively called short-fin and long-fin current feeders for convenience. The other one was a planar current feeder. Each of the fin-type current feeders had five fins with a thickness of $0.9 \mathrm{~mm}$. Because the fluidized bed length in our experiments never exceeded $5.5 \mathrm{~cm}$, about half the surface area of the current feeder was masked to minimize the area exposed directly to the electrolytes. A copper wire, functioning as the reference electrode, was placed above particle beds near the membrane. A potentiostat/galvanostat, HA320 from Hokuto Penko, Limited, was used to supply electric power and to control potentials between working and reference electrodes.

Experimental procedures.-Steady-state polarization curves of the fluidized bed electrodes with three types of current feeders were measured at various Reynolds numbers ( $\left.\operatorname{Re}=v d_{\mathrm{p}} / \nu\right)$, which is based on the particle's diameter $d_{\mathrm{p}}$, fluid superficial velocity $v$, and kinematic viscosity $v$. The electrolytes were circulated from a reservoir of $40 \mathrm{li}$ ters, in which the variation of the concentration can be ne-

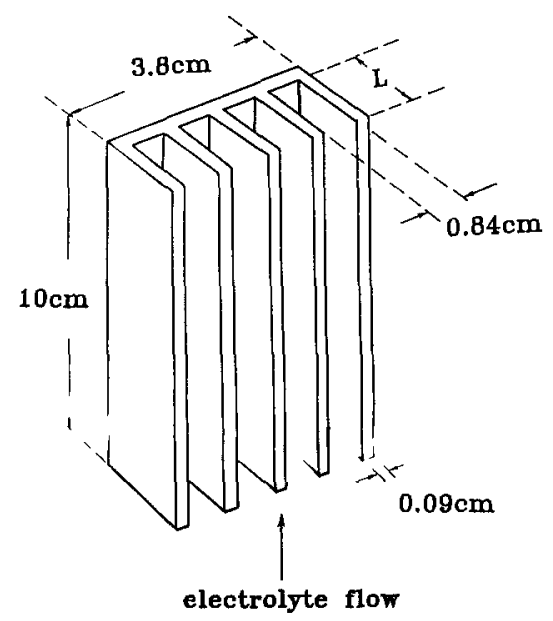

Fig. 2. The fin-type current feeder, $L=0,0.7$, or $1.2 \mathrm{~cm}$. 
glected. The circulating operation was carried out at a controlled potential, and concentration of cupric ion in the reservoir was determined by a spectrophotometer (Miltinroy Company, Spectronic 601). Wavelength was set on $810 \mathrm{~nm}$. Copper particles were treated with acetone and diluted nitric acid before being used, and the catholytes in the reservoir were purged by high-purity nitrogen before and during the experiments.

\section{Results and Discussion}

Polarization curves.-Polarization curves of fluidized bed electrodes with the planar current feeder measured at various Reynolds numbers $\left(R e=v d_{\mathrm{p}} / v\right)$ are shown in Fig. 3. The current densities calculated in all figures are based on the exposed surface area of the planar current feeder, $12.16 \mathrm{~cm}^{2}(3.2 \times 3.8 \mathrm{~cm})$, which is covered by copper particles before being fluidized (the initial height of bed: $3.2 \mathrm{~cm}$; width: $3.8 \mathrm{~cm}$ ). The concentration of cupric ion used in the experiments was $0.02 M$. The parameter $\epsilon$, appearing in Fig. 3, represents the porosity of the fluidized bed and the unfluidized bed porosity was measured at 0.668. As shown in Fig. 3, the current densities with the planar current feeder increase with the applied potentials. There is no limiting-current plateau observed, i.e., the current flows in the fluidized bed electrodes at the range of applied voltages are not controlled by mass transfer. The same result was also obtained by Fleischmann and Kelsall (13), who explained that nonuniform potential distribution arose from the low effective conductivity of fluidized bed electrodes. Thus, polarization curves were almost linear in this situation. A remarkable result can be seen in Fig. 3 in that the current densities of polarization curves do not rise as increasing Reynolds numbers $\left(\operatorname{Re}=v d_{\mathrm{p}} / v\right)$, which can be related to bed expansions (i.e., the ratio of increased bed length by fluidization to the initial bed length). The same result is also observed in Fig. 4, which shows polarization curves of fluidized bed electrodes with the short-fin current feeder. But as shown in Fig. 5, the current densities of polarization curves with the long-fin current feeder are raised higher as increasing Reynolds numbers or bed expansions. It has to be pointed out here that it can further be operated at bed expansions higher than $48 \%$. It is found that the long-fin current feeder offers the best performance.

Effects of bed expansion.-It can be found in Fig. 3-5 that the long-fin current feeder gives higher current densities at the same applied potential, and the short-fin current feeder does not offer obvious enhancement in current densities at the same condition. Figure 6 shows current densities $v s$. bed expansions for these three current feeders at an applied voltage of $-0.8 \mathrm{~V}$, and there is a significant difference

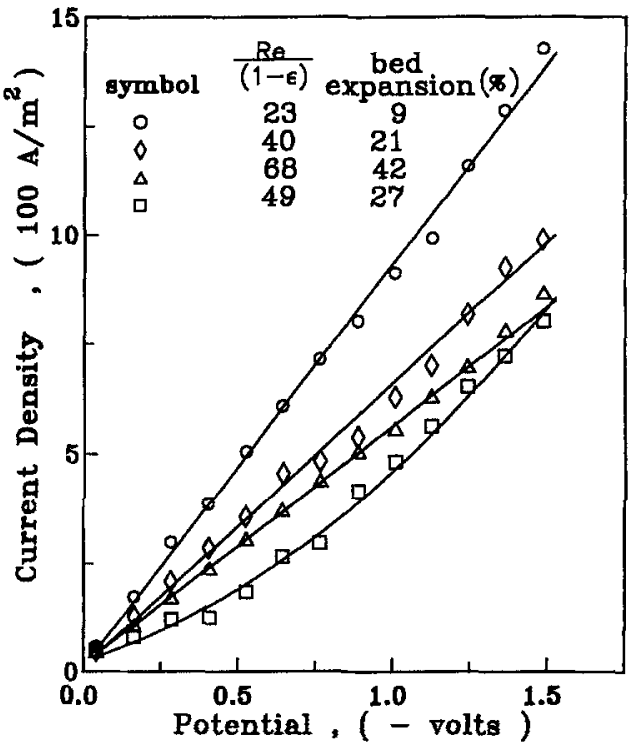

Fig. 4. Polarization curves with the short-fin current feeder.

of deposition rates between the long-fin current feeder and the other two. As shown in Fig. 6, the current densities decrease as bed expansions increase by using the planar and short-fin current feeders, but the current density by the short-fin current feeder is higher than that by the planar current feeder, and asymptotic current densities seem to be reached at higher bed expansions. These asymptotic current densities seem to be contributed mostly from the particles near the surface of the current feeders, because the ratio of surface area of current feeders, short-fin to planar, is 2.47 , and the ratio of asymptotic current densities short-fin to planar, is 1.5. Furthermore, our experimental results (23) indicated that the limiting current densities of a fluidized cell with inert particles, which using the planar and short-fin current feeders, were in the range of $70-100 \mathrm{~A} / \mathrm{m}^{2}$ and $100-200 \mathrm{~A} / \mathrm{m}^{2}$, respectively; they were close to the asymptotic current densities. The current densities with the long-fin current feeder are raised as increasing bed expansions, and they are much higher than those of the other two types.

The contribution of metal deposition on the current feeder itself could also be investigated. In our experimental results the contribution of the long-fin current feeder itself to the total current was measured by the anodic dissolution of the current feeder individually after the metal deposition was completed. It was found that less than $5 \%$

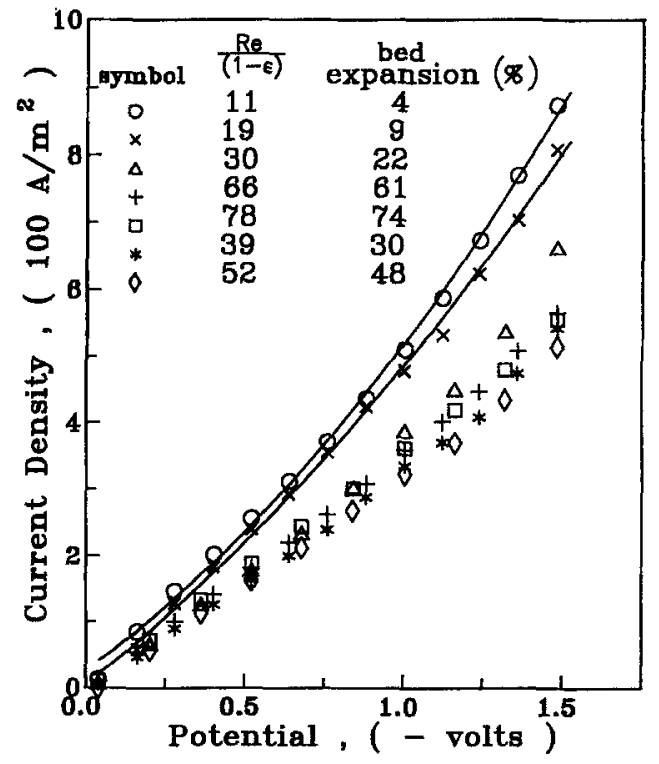

Fig. 3. Polarization curves with the planor current feeder.

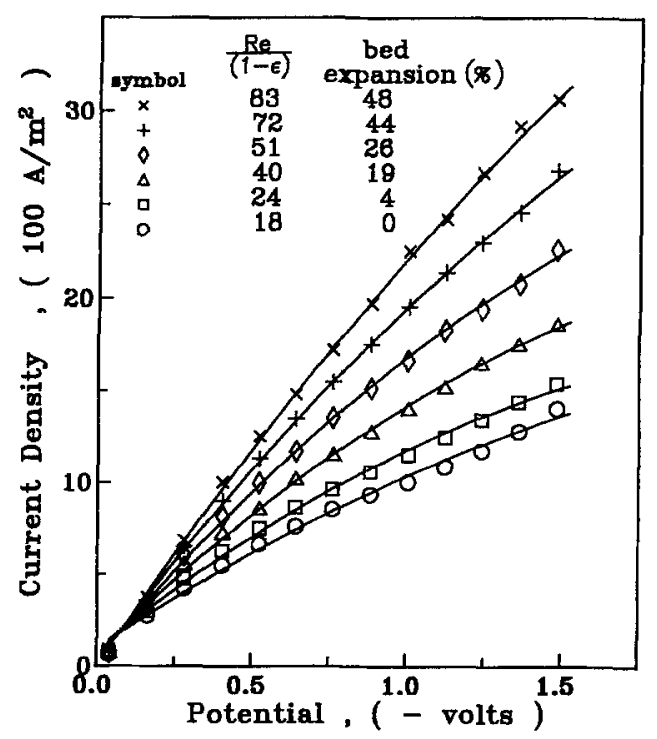

Fig. 5. Polarization curves with the long-fin current feeder. 


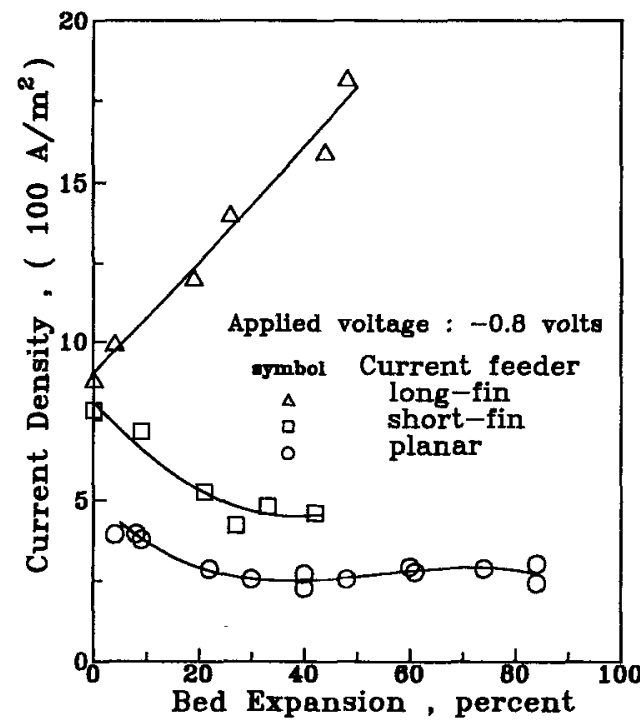

Fig. 6. Effects of bed expansion on current density for various current feeders.

of the metal deposition was deposited at the surface of the long-fin current feeder, and it can be concluded that the current enhanced obviously by the fin-type current feeder was mostly contributed by the metal deposition on the fluidized particles.

Because bed expansions can greatly affect the effective conductivity of the particle phase, which will be decreased by rising bed expansions, it can be regarded that while it behaves in this way the charge-transfer rate in the solid phase dominates current flows in fluidized bed electrodes. The other behavior is that current densities are increased as Reynolds numbers increase, and both the behavior of fluidized bed electrodes with the long-fin current feeder and packed bed electrodes belong to this case. This reveals that mass transfer perhaps dominates current flows in the fluidized bed cells with the long-fin current feeder.

Copper recovery experiments.-Since the polarization curves reveal that a suitable fin-type current feeder will greatly enlarge the current densities, it is appropriate to verify the feeder in its performance in metal recovery. The performance of a cell removing copper ions from the dilute solution with three types of current feeders is shown in Fig. 7. The potential was controlled at $-1.0 \mathrm{~V}$, and the bed expansions were operated at 25,50 , and $75 \%$ respectively. The volume of the reservoir was 10 liters, and $132 \mathrm{~g}$ of cop-

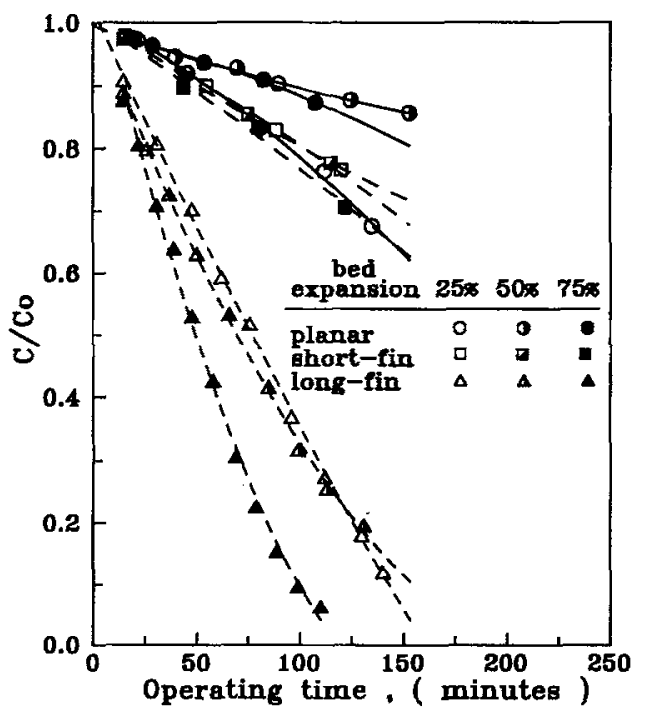

Fig. 7. Comparisons between the three current feeders in performance of metal recovery (initial concentration: $350 \mathrm{ppm}$; applied potential: $-1 \mathrm{~V})$.

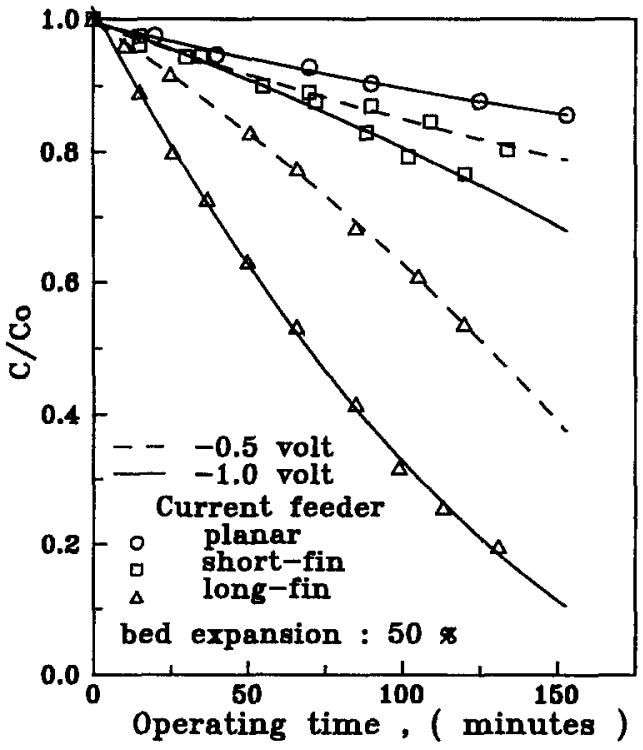

Fig. 8. Effects of applied voltage on the performance of metal recovery (initial concentration: $\mathbf{3 5 0} \mathrm{ppm}$ ).

per particles were used as cathodes. The initial concentration of the cupric ion was $350 \mathrm{ppm}$. Figure 7 shows that the fin-type current feeder actually enhances the performance of fluidized bed electrodes in metal recovery. After 100 min of operation with $75 \%$ bed expansion, the concentration of cupric ion in the reservoir was reduced to $10 \%$ of the initial concentration, $350 \mathrm{ppm}$, by using the long-fin current feeder. The concentration of cupric ion was reduced to 88 and $76 \%$ of the initial concentration while using the planar and short-fin current feeders, respectively. Figure 7 indicates that the experimental curves of the same type of current feeders are very close to each other, and they can be classified into three groups by various current feeders. The concentration curves by using the long-fin current feeder are lowest in Fig. 7, and it means that the long-fin current feeder gives a better performance in metal recovery.

The effect of applied voltage on the performance of fiuidized bed electrodes in metal recovery is depicted in Fig. 8. The concentration decrease with time by the longfin current feeder at $-0.5 \mathrm{~V}$ is less than that at $-1.0 \mathrm{~V}$, but more than that by the short-fin current feeder at -1.0 and $-0.5 \mathrm{~V}$. Furthermore, the performance by the short-fin current feeder at $-0.5 \mathrm{~V}$ is better than that by the planar current feeder at $-1.0 \mathrm{~V}$. Because the applied voltage is lowered by using the fin-type current feeder to achieve the same level of performance in metal recovery, the fin-type current feeders can also achieve some saving of electric energy.

Discussion.-The fin length of the long-fin current feeder is three-fifths of the thickness of the cathodic reaction part in the cell; longer fins seem to favor current flows in fluidized bed electrodes, which have been verified by our experimental results. This may be explained by considering the charge transfer process as the conductive mechanism, in which contacts between particles and current feeders are very important. As has been described, aggregated particles contact with current feeders to form monopolar particles where metal electrodeposition occurs. As the possibility of contacts between particles and current feeders is raised, the ratio of monopolar particles in the fluidized bed will be increased, and the current will be enhanced. The fin-type current feeder would increase the possibility of contacts between fluidized particles and the current feeder; the deposition rate could be enhanced in this way. Nonuniform fluidization was observed at high bed expansions in our experiments, while the long-fin current feeder was used. Partial particles circulated in the bed, especially at the top of the bed. The circulation of the particles may have enhanced the contact frequency between the particles and the current feeder. 
Although the ratio of surface area, long-fin to planar, is 3.53 , it produces higher enhancement ratio corresponding to the ratio of the surface area. It is not enough to explain the current enhancement caused by the long-fin current feeders, if it is based on contact frequency, especially at higher bed expansions. It may be explained further that reaction rates which occurred on the particle's surface are dependent on positions in the beds. When fins are extended toward the membrane, particles near the surface of the current feeder have more opportunities to become monopolar, and their potentials are very close to the current feeder. Like flow-by packed bed electrodes, current densities increase in the direction toward the membrane (4), i.e., the particles near the membrane are more electrochemically active. So the deposition rates will be enhanced more, even though the surface area of the long-fin current feeder has not increased correspondingly. Using the short-fin current feeder has also enlarged the current, but with less enhancement. It may be explained that less monopolar particles are formed by short-fin current feeders, and are still far from the membrane, due to shorter extended fins. The long-fin current feeder could produce more monopolar particles, which also have more electrochemical activity than the short-fin or planar current feeder. Thus, the enhancement of current densities by the long-fin current feeder is much better than by the other two types of current feeders.

\section{Conclusion}

From the polarization and circulation experiments, it is found that fin-type current feeders could enhance the metal deposition rates in fluidized bed electrodes. The relationship between current density and bed expansion is also altered by increasing the fin length. Current densities are decreased as bed expansions are raised when using planar as well as short-fin current feeders. But on the contrary, by using the long-fin current feeder, the current density is enhanced with increasing bed expansions, and the performance of metal recovery is improved. In addition to the enhanced metal recovery, the fluidized bed electrodes with an appropriate fin-type current feeder can also allow a more flexible range of bed expansions. The enhancement of current feeders with fins extended can be explained by the fact that it has not only increased the contact probability between particles and current feeders, but has also in- creased numbers of more electrochemically active monopolar particles in the cell.

Manuscript submitted Nov. 1, 1991, revised manuscript received Feb. 18, 1991.

National Taiwan University assisted in meeting the publication costs of this article.

\section{REFERENCES}

1. M. Fleischmann, F. Goodridge, and J. R. Backhurst, Brit. Pat., 1,194,181 (1970).

2. F. Coeuret, P. le Goff, and F. Vergnes, French Pat, $1,500,269$ (1967).

3. D. N. Bennion and J. Newman, J. Appl. Electrochem., 2, 113 (1972).

4. M. A. Enriquez-Granados, D. Hutin, and A. Storck, Electrochim. Acta, 2\%, 303 (1982).

5. T. Berent, I. Fells, and R. Mason, Nature, 223, 1054 (1969).

6. J. R. Backhurst, F. Goodridge, R. E. Plimley, and M. Fleischmann, ibid., 221, 55 (1969).

7. C. Oloman and A. P. Watkinson, Can. J. Chem. Eng., 53,268 (1975).

8. F. Goodridge, C. J. H. King, and A. R. Wright, Electrochim. Acta, 22, 1087 (1977).

9. F. Goodridge and C. J. Vance, ibid., 24, 1237 (1979).

10. F. Coeuret, J. Appl. Electrochem., 10, 687 (1980).

11. S. Germain and F. Goodridge, Electrochim. Acta, 21, 545 (1976).

12. R. L. Leroy, ibid., 23, 815 (1978).

13. D. Hutin and F. Coeuret, J. Appl. Electrochem., 7, 463 (1977).

14. M. Fleischmann and G. H. Kelsall, ibid., 14, 269 (1984).

15. B. J. Sabacky and J. W. Evans, Metall. Trans., 8B, 5 (1977).

16. F. Goodridge, Electrochim. Acta, 22, 929 (1977).

17. M. Fleischmann and J. W. Oldfield, J. Electroanal. Chem., 29, 211 (1971).

18. M. Fleischmann and J. W. Oldfield, ibid., 29, 231 (1971).

19. R. E. Plimley and A. R. Wright, Chem. Eng. Sci., 39, 395 (1984).

20. T. Huh and J. W. Evans, This Journal, 134, 308 (1987).

21. J. K. Lee, L. W. Shemilt, and H. S. Chun, J. Appl. Electrochem, 19, 877 (1989).

22. T. Huh and J. W. Evans, This Journal, 134, 317 (1987).

23. S. C. Yen and C. Y. Yao, Unpublished results.

24. K. Kusakabe, S. Morooka, and Y. Kato, J. Chem. Eng. Jpn., 14, 208 (1981).

\title{
An Investigation into Membrane Potentials Generated Across Mullite in Mixtures of Molten Alkali Metal Bromides
}

\author{
M. L. Orfield ${ }^{\star}$ \\ Department of Chemistry, University of Wisconsin-Stout, Menomonie, Wisconsin 54751
}

\begin{abstract}
Mullite functions as an ionic conductor of alkali metal cations at high temperatures. The membrane potentials of two mullite membranes, MV30 and MV33, were measured in mixtures of the alkali metal cations. These potentials were predicted by the equations used to predict membrane potentials in aqueous systems. The potential selectivity coefficient of MV30 for $\mathrm{Na} / \mathrm{K}, \mathrm{Li} / \mathrm{K}$, and $\mathrm{Na} / \mathrm{Li}$ were calculated and the potential selectivity coefficient of $\mathrm{MV} 33$ for $\mathrm{Na} / \mathrm{K}$ was evaluted. The range of composition over which the behavior of each mullite may be predicted was discussed.
\end{abstract}

Glassy membranes, sensitive to a single ion, form the basis for many separators and ion selective electrodes in use in aqueous systems. The success of such electrodes depends upon an understanding of the relationship between the composition of the solution and the potential generated across the membrane. In aqueous systems this relationship has been thoroughly examined and exploited to prepare glasses selective for a variety of ions.

* Electrochemical Society Active Member.
Since glassy membranes are known to conduct ions well in molten salt solutions, it seems likely that they may obey the same kind of mathematical relationships applicable in aqueous systems. A few investigators have explored this area. Doremus measured the potentials across silica and Pyrex glasses in molten nitrates $(1,2)$. Notz and Keenan also measured potentials across Pyrex in molten nitrates $(3,4)$. van Reenan et al. and Stern investigated potentials across silica in molten chlorides to a limited extent (5-7). However potentials across these glasses could not be 